\title{
Violência Doméstica e Risco para Problemas de Saúde Mental em Crianças e Adolescentes
}

\author{
Domestic Violence and Risk for Mental Health \\ in Childhood and Adolescence
}

\author{
Natália Amaral Hildebrand*, Eloisa Helena Rubello Valler Celeri, André Moreno Morcillo \\ \& Maria de Lurdes Zanolli \\ Universidade Estadual de Campinas, Campinas, SP, Brasil
}

\begin{abstract}
Resumo
A literatura demonstra que sofrer violência doméstica na infância e juventude é um risco para o desenvolvimento das psicopatologias. Este estudo utilizou o Strenghts and Difficulties Questionnarie (SDQ) e encontrou prevalência de $65,5 \%$ de possibilidades de problemas de saúde mental em crianças e adolescentes (4-16 anos), vítimas de violência doméstica, acompanhados em Serviços de Referência, todos residentes em um município brasileiro. O resultado encontrado foi considerado elevado, se comparado à população geral. A violência doméstica foi observada como fator de risco para problemas de saúde mental nas crianças e adolescentes estudados, agravada pelo uso preocupante de bebida alcoólica pelo responsável, ou este estar fora do processo produtivo. Não foram encontrados fatores de proteção efetivos, principalmente em relação à rede de proteção. Esses resultados sinalizam a necessidade de implementação de mecanismos que avaliem a efetividade de programas específicos nas áreas de saúde mental e violência doméstica.

Palavras-chave: Saúde mental, prevalência, fator de risco e proteção, violência doméstica, SDQ.

Abstract

Literature shows that experiencing domestic violence in childhood and young adulthood is a risk for the development of psychopathology. This study used the Strengths and Difficulties Questionnaire (SDQ) and found a prevalence of $65.5 \%$ of possibility of mental health problems in children and adolescents (4-16 years old) who were victims of domestic violence followed in Referral Services in a Brazilian city. The result found was considered high when compared to the general population. Domestic violence was observed as a risk factor for mental health problems among the studied children and adolescents, aggravated by caregivers' alcoholism or unemployment. No effective protective factors were found, mainly regarding the protection network. These results indicate the need for reassessing the effectiveness of specific programs in the areas of mental health and domestic violence. Keywords: Mental health, prevalence, risk and protective factor, domestic violence, SDQ.
\end{abstract}

A violência é um fenômeno presente nos diversos âmbitos da vida, tratando-se, portanto, de um problema social, de prática política e relacional da humanidade. A violência social é a terceira maior causa de mortalidade na população geral, e a primeira entre os adolescentes e crianças a partir de um ano. Apesar de as crianças e adolescentes serem suas mais frequentes vítimas, é difícil avaliar com precisão a incidência da violência, uma vez que os estudos sobre o tema, nos diversos países, encontram-se em diferentes etapas. Isso ocorre principalmente pelas dificuldades na coleta de dados e na realização de denúncias que envolvam a temática da violência, fatores esses que interferem na realização de estimativas seguras (Abranches \& Assis, 2011; Assis, Avanci, Pesce, \& Ximenes, 2009; Azevedo \& Guerra, 2005; Ministério da Saúde, 2008).

Nesta pesquisa tratou-se especificamente do tema violência doméstica contra crianças e adolescentes (VDCCA). Este é um fenômeno que traz à tona a questão da agressividade no ser humano, e levanta reflexões sobre

\footnotetext{
* Endereço para correspondência: Departamento de Pediatria, Faculdade de Ciências Médicas, Universidade Estadual de Campinas, Rua Tessália Vieira de Camargo, 126, Cidade Universitária "Prof. Zeferino Vaz", Campinas, SP, Brasil 13083-887. Fone: (19) 3521-7824/7193 / 7322 / 7111. E-mail: nataliaah@gmail.com, evaller@ fcm.unicamp.br, andre.morcillo@gmail.com e zanolli@ fcm.unicamp.br
}

Agradecemos o apoio financeiro da FAPESP (Fundação de Amparo a Pesquisa do Estado de São Paulo - Brasil) a essa pesquisa.

Este estudo foi aprovado pelo Comitê de Ética em Pesquisa da Faculdade de Ciências Médicas da Universidade Estadual de Campinas - UNICAMP (parecer 173/2010) e também pela Secretaria Municipal de Cidadania e Inclusão Social do Município de Campinas. Os sujeitos e os seus responsáveis concordaram em participar da pesquisa. 
suas causas e consequências. Entre as consequências está a possibilidade de transtorno mental dos sujeitos vítimas de violência.

A Organização Mundial de Saúde (OMS, Word Health Organization [WHO], 1999) define como maus tratos, todas as formas de abuso ou violência (física, emocional ou psicológica, sexual, negligência, exploração comercial ou outra), no contexto de relação de responsabilidade, confiança ou poder do adulto em relação à criança ou ao adolescente. Maus tratos podem causar dano real ou potencial ao desenvolvimento, capacidade de sobrevivência, saúde ou dignidade aos sujeitos que sofrem violência. Esse é um fenômeno que se reproduz a partir de um ciclo, podendo a criança que sofre violência doméstica, ou maus tratos, repeti-lo em diferentes situações (Walker, 1979).

Os tipos mais comuns de violência doméstica contra crianças e adolescentes são: Violência Física: dano físico ou lesão causada ao sujeito pelo uso da força, auxílio de armas ou instrumentos; Violência Sexual: toda ação na qual uma pessoa, a partir do estabelecimento de uma relação de poder, obriga outra à realização de práticas sexuais, utilizando força física, influência psicológica e uso de armas ou drogas; Violência Psicológica: toda ação ou omissão que possa ocasionar prejuízos ou danos à autoestima, identidade ou ao desenvolvimento biopsicossocial do sujeito, e Negligência ou abandono: omissão de responsabilidade de um ou mais membros da família em relação a outro, sobretudo àqueles que necessitam de cuidados, atenção ou orientação, em função da idade ou determinada condição física, permanente ou temporária (Day et al., 2003).

A maioria dos estudos aborda a temática da VDCCA, a partir do que se observa como causas e, também, as consequências físicas nos sujeitos. Alguns apontam possíveis associações entre vivenciar situações de maus tratos na infância e juventude aos riscos que elas possam representar para o desenvolvimento de transtornos mentais, em cuja ocorrência, verifica-se o aparecimento de sinais e sintomas de ordem emocional como resposta do sujeito aos estressores físicos e psicossociais. O estudo dessas possíveis associações tem sido de grande importância para as pesquisas em psicopatologia (Assis et al., 2009; FleitlichBilyk \& Goodman, 2001; A. Goodman, Fleitlich-Bilyk, Patel, \& Goodman, 2007; Paula et al., 2008; Polanczyk, Zavaschi, Benetti, Zenker, \& Gammerman, 2003; Serafim, Saffi, Achá, \& Barros, 2011).

Em relação aos dados sobre a saúde mental infanto-juvenil, a OMS (WHO, 2005) e o Ministério da Saúde do Brasil (2005) estimam que de $10 \%$ a $20 \%$ da população de crianças e adolescentes sofram de algum transtorno psiquiátrico. Alguns autores, em estudos internacionais populacionais, encontraram grande variabilidade de problemas, com prevalência estimada de $1 \%$ a $51 \%$ (Assis et al., 2009). A maioria dos estudos realizados em países em desenvolvimento, como o Brasil, aponta prevalência entre $10 \%$ e $20 \%$, sendo que alguns encontraram risco em torno de 30\% (Canino et al., 2004; Fleitlich-Bilyk \&
Goodman, 2004; Paula, Duarte, \& Bordin, 2007). Gallo et al. (2011), em um estudo brasileiro com crianças de 11 anos, utilizando-se o Questionário de Capacidades e Dificuldades (SDQ), encontraram prevalência de $32 \%$ de possibilidades de problemas de saúde mental. Foram observados os seguintes fatores de risco para tais transtornos: características maternas, fatores socioeconômicos e biológicos da genitora e da criança.

Nos poucos estudos dos quais se dispõe, quanto à prevalência de possíveis transtornos psiquiátricos associados a situações de VDCCA, a maior parte é com vítimas de violência sexual. Nessas pesquisas, verificaram-se índices de depressão que variaram entre $38,6 \%$ a $59,2 \%$, e de transtorno de estresse pós-traumático (TEPT), de 29,3\% a 69\% (Lorion \& Saltzman, 1993; Serafim et al., 2011). Os estudos que correlacionam os temas violência e saúde mental ainda se encontram no início e, por isso, apresentam muitas diferenças metodológicas que dificultam a comparação entre os dados (Assis et al., 2009).

Apesar dessas questões, verificam-se avanços nessas áreas temáticas, os quais apontam para a necessidade de mais pesquisas sobre fatores de risco (vulnerabilidade) e de proteção para o desenvolvimento das psicopatologias infanto-juvenis (Cicchetti \& Valentino, 2006). Os fatores de risco e de proteção não se constituem como conceitos diferentes, podendo ser considerados como polos, positivo e negativo, de uma mesma questão. A essência de ambos é que precisam estar associados a variações em relação aos riscos ou às condições de vulnerabilidade. Os fatores de risco podem levar a reações, como a falta de adaptação no que se refere às áreas de desenvolvimento do indivíduo; enquanto que os fatores de proteção teriam a função de atenuar as situações de vulnerabilidade, reduzindo o risco. Características individuais, como a personalidade, níveis de autoestima, sociabilidade, inteligência, entre outras, e/ ou características ambientais, como a coesão familiar, presença de figuras significativas ou sistemas de suporte externo, possibilitam a vivência de situações adversas de maneira positiva, quando presentes; ou negativa, quando ausentes (Rutter, 1987; Werner, 1989).

$\mathrm{O}$ atendimento e proteção às pessoas que tiveram seus direitos violados, como no caso da violência doméstica, estão sob a responsabilidade do Ministério de Desenvolvimento Social e Combate à Fome. Com a deliberação pela implantação do Sistema Único de Assistência Social (SUAS), em 2003, os serviços socioassistenciais passaram a ser organizados a partir do nível de complexidade, subdividindo-se em: Proteção Social Básica (prevenção) e Proteção Social Especial (proteção). O CREAS (Centro de Referência Especializado de Assistência Social) tratase de um órgão público que realiza o acompanhamento psicossocial de famílias em situação de risco por violação de seus direitos (Ministério do Desenvolvimento Social e Combate à Fome, 2005, 2010).

Neste estudo, foi realizada uma avaliação dos possíveis problemas de saúde mental em crianças e adolescentes vítimas de violência doméstica, atendidos em Serviços 
Hildebrand, N. A., Celeri, E. H. R. V., Morcillo, A. M. \& Zanolli, M. L. (2015). Violência Doméstica e Risco para Problemas de Saúde Mental em Crianças e Adolescentes.

Especializados de Referência, com o objetivo de determinar sua prevalência, realizar a caracterização da população estudada e analisar fatores de risco e de proteção para o desenvolvimento das psicopatologias nesses sujeitos.

\section{Método}

\section{Participantes}

Participaram deste estudo de delineamento transversal descritivo e analítico, realizado nos anos de 2010 e 2011, 252 crianças e adolescentes vítimas de violência doméstica, atendidos em Serviços Especializados conveniados ao CREAS de um município brasileiro, sorteados aleatoriamente, mantendo-se a proporção entre as faixas etárias do total de crianças e adolescentes atendidos no serviço (4-7 anos, 8-11 anos e 12-16 anos) e por região do município (Norte, Sul, Leste, Sudoeste e Noroeste). O cálculo amostral inicial foi de 156 sujeitos, e levou-se em consideração a prevalência média de, no mínimo, $30 \%$ de possibilidades de problemas de saúde mental, de acordo com a revisão de literatura (Canino et al., 2004; Lorion \& Saltzman, 1993; Serafim et al., 2011). Com o decorrer do estudo, a prevalência encontrada mostrou-se muito superior à estimativa inicial, e um novo cálculo amostral foi realizado, ampliando-se para 252 sujeitos.

Os Serviços Especializados são entidades psico-socioassistenciais, que funcionam em parceria com o governo municipal, por meio do CREAS, para o atendimento de famílias em situação de violação de direitos. $\mathrm{O}$ acompanhamento das famílias é realizado por uma dupla de profissionais (um psicólogo e um assistente social), com o objetivo de minimizar as situações de violência e também de promover a proteção das crianças e adolescentes.

Foram excluídos os sujeitos com diagnóstico prévio, de transtorno neurológico ou retardo mental, realizado por serviços de saúde.

\section{Instrumentos}

Para coleta dos dados, foi utilizado o "Questionário de Capacidades e Dificuldades" (SDQ), na versão brasileira validada (Fleitlich-Bilyk \& Goodman, 2004), do Strenghts and Difficulties Questionnaire (R. Goodman, 1997, 2001). O SDQ é um instrumento que avalia problemas de saúde mental infanto-juvenil, de sujeitos na faixa etária de 4-16 anos. É apresentado em três versões: de autoaplicação (1116 anos), de pais e a de professores (4-16 anos), e composto por 25 questões, divididas em cinco subescalas, tendo cada uma delas cinco questões: problemas emocionais, de conduta, hiperatividade, relacionamentos interpessoais e comportamento pró-social. As opções de escolha das respostas são: falso, verdadeiro e mais ou menos verdadeiro; e cada item recebe uma pontuação específica de 0 a 10 pontos. O SDQ tem um adendo que avalia o impacto dos sintomas, somado à pontuação final. A somatória das subescalas permite a classificação em três categorias quanto à saúde mental: desenvolvimento normal $(\mathrm{N})$, limítrofe (L) e anormal (A). Quanto maior a pontuação, maior é a possibilidade de problemas. Este mesmo critério pontua cada uma das subescalas e, assim, permite analisar o tipo de sintoma apresentado, com exceção da categoria pró-social (quanto maior o resultado, melhor é a capacidade de desenvolvimento das potencialidades do indivíduo). Este é um instrumento de fácil aplicação e dirigido à população geral (Duarte \& Bordin, 2000; Fleitlich-Bilyk, Cortazar, \& Goodman, 2000; R. Goodman, 1997, 2001).

De acordo com as instruções de interpretação do SDQ (http://www.sdqinfo.com/), pelo menos $80 \%$ das crianças na comunidade devem ser N, 10\% L e 10\% A. Em amostra de baixo risco, são considerados "casos", apenas quando se tem alta pontuação em uma das quatro escalas de dificuldades. Neste estudo, a população foi considerada de alto risco em decorrência da violência doméstica; portanto, ao se realizar a avaliação de problemas de saúde mental foram consideradas, como recomendado, as categorias $\mathrm{Le}$ A (R. Goodman, 1997, 2001). Foram utilizadas as versões para responsáveis das crianças de 4-10 anos e a versão de autoaplicação de 11-16 anos.

Os fatores de risco e proteção analisados neste estudo foram baseados em dados da literatura (Fleitlich-Bilyk \& Goodman, 2001; A. Goodman et al., 2007; Koller \& Lisboa, 2007; Poletto, Koller, \& Dell'Aglio, 2009; Rutter, 1987; Werner, 1989) e reafirmados pelos profissionais dos Serviços Especializados. Foram estudados fatores de risco: em relação aos responsáveis ou à família (conflitos familiares; problemas de saúde mental; violência de gênero; uso preocupante de substâncias psicoativas SPA; envolvimento com tráfico de drogas/ criminalidade; prostituição; uso preocupante de bebida alcoólica; doença crônica ou questões de saúde; ameaça de morte de alguém da família; se alguém da família encontrava-se foragido ou recluso e se a família estava vivendo em situação de rua ou mendicância) e, em relação às crianças e adolescentes (abrigamento institucional, se já fora abrigado em algum momento na vida; evasão escolar; gravidez na adolescência, e estar inserido em medida socioeducativa). Foram analisados como possíveis fatores de proteção: participação em atividades socioculturais/esportivas ou comunitárias (tanto a criança/adolescente quanto os responsáveis); situação trabalhista dos responsáveis; participação em atividades religiosas; escolaridade dos responsáveis; morar com guardiões legais para proteção contra situações de violência e contar efetivamente com apoio dos Serviços/Rede de Proteção, como sistemas de suporte externos. Foram considerados fazendo parte desses sistemas: saúde, educação, serviços de assistência social, cultura, esporte, habitação, trabalho e renda e os Sistemas de Garantia de Direitos - SGD (Conselho Tutelar, Vara da Infância e Juventude, Ministério Público, Delegacia de Defesa da Mulher, Delegacia da Infância e Juventude).

Os dados para caracterização, contextualização da população e para identificar os possíveis fatores de risco e proteção, foram coletados por diferentes psicólogos e assistentes sociais, em prontuários de acompanhamento psicossocial dos Serviços Especializados. Apesar de isso poder 
ser uma limitação, deve-se ressaltar que, para identificação desses dados, as fichas de anamnese foram padronizadas pelos serviços e muitas das informações foram coletadas ao longo do processo de acompanhamento psicossocial, por meio de entrevistas abertas e semiestruturadas. Famílias em situação de violência doméstica apresentam dificuldades de vinculação e, também, de revelação de determinados dados. Optou-se por essa forma de coleta dos dados, em função do tipo de vínculo já estabelecido entre as famílias e os profissionais.

\section{Procedimentos}

A aplicação do instrumento foi realizada em um encontro com cada sujeito selecionado (com um dos responsáveis, no caso de crianças de 4 a 10 anos, e com o próprio adolescente de 11 a 16 anos). Com o objetivo de se evitar dificuldades na compreensão dos questionários, por parte dos entrevistados, os entrevistadores foram os profissionais que atendiam à família e com quem o sujeito havia estabelecido vínculo mais significativo (o psicólogo ou o assistente social). Cada entrevista foi realizada no Serviço Especializado que acompanhava a família, no dia agendado para o atendimento, com consentimento dos participantes e dos responsáveis. Os sujeitos que apresentaram dificuldade na compreensão para responder ao instrumento foram excluídos no momento da entrevista.

Uma das dificuldades encontradas foi o alto índice de absenteísmo na entrevista de aplicação do instrumento, pois 80 sujeitos $(31,7 \%)$ não compareceram, sendo $25 \%$ na faixa etária de 4-7 anos, 32,5\% de 8-11 anos e 42,5\% de 12-16 anos. Foi necessário substituí-los após três tentativas de aplicação. A não adesão aos atendimentos e ao acompanhamento no Serviço Especializado trata-se de uma característica da população estudada, que pode estar associada às situações de violência doméstica, aos outros fatores de risco presentes nestas famílias e, também, ao formato de como os programas de atendimento foram estruturados e desenvolvidos.

Este estudo foi aprovado pelo Comitê de Ética em Pesquisa da Universidade (Parecer $n^{\circ}$ 173/2010) e pela Secretaria Municipal de Cidadania e Inclusão Social do Município. Os responsáveis concordaram e assinaram o Termo de Consentimento Livre e Esclarecido.

\section{Análise dos Dados}

$\mathrm{Na}$ análise dos dados foram empregados os programa Statistical Package for Social Sciences, versão 16.0 (SPSS Inc., Chicago, IL, EUA) e o Epi-Info, versão $6.04 \mathrm{~b}$ - WHO. Determinou-se a prevalência e seu Intervalo de Confiança de 95\%-IC95\% (método exato pela distribuição binomial) de problemas de saúde mental, em relação ao sexo e grupos etários. Para avaliação da associação entre problemas de saúde mental e as demais variáveis (fatores de risco e proteção, faixa etária e tipo de violência), empregou-se o teste do Qui-quadrado ou o Teste Exato de Fisher. Em todos os casos, adotou-se o nível de significância de 5\%.

\section{Resultados}

Serão apresentados inicialmente: a caracterização geral da população em relação a sexo e idade; aspectos socioeconômicos e culturais da família: renda mensal, situação trabalhista, escolaridade dos responsáveis e atividade religiosa; configuração familiar e caracterização da violência. Em seguida, serão descritas as prevalências de possibilidades de problemas e sintomas de saúde mental, nos sujeitos estudados, e analisadas as variáveis como possíveis fatores de risco e de proteção para o desenvolvimento de problemas de saúde mental em vítimas de violência doméstica.

Das 252 crianças e adolescentes que participaram do estudo, 52,8\% eram do sexo feminino. Em relação à faixa etária, observou-se que as crianças entre 4-7 anos corresponderam a $24,6 \%$, as de $8-11$ anos a $32,9 \%$ e os adolescentes de 12-16 anos, 42,5\%.

Em relação à renda mensal em salários mínimos - SM (em Reais, no valor de $\mathrm{R} \$ 622,00), \mathbf{8 0 , 5} \%$ das famílias recebiam de 0 até $2 \mathrm{SM}$, incluindo nessas rendas benefícios sociais, já que $68,3 \%$ das famílias contam com esse auxílio. Em relação ao restante, 13,5\% recebiam de 2 a 3 SM e apenas $6 \%$ mais de $3 \mathrm{SM}$.

Quanto à situação trabalhista dos responsáveis, a minoria destes encontrava-se na categoria Trabalho Formal $(37,8 \%)$, comparado à somatória das outras categorias (62,2\%): 28,7 \% Não Trabalham/ Desempregados, 28,3\% Trabalho Informal e 5,2\% Aposentados/Recebem BPC Benefício de Prestação Continuada.

Quanto à escolaridade dos responsáveis, verificou-se que 58,3\% deles cursaram apenas o Ensino Fundamental (EF), sendo $28,6 \%$ do $1^{\circ}$ ao $5^{\circ}$ ano do EF/ supletivo ( $5^{\circ}$ Ano EF) e $29,7 \%$ do $5^{\circ}$ ao $9^{\circ}$ ano do EF. Em relação ao restante dos responsáveis, $27,8 \%$ cursaram ensino médio, curso técnico ou superior e 13,9\% são não alfabetizados/ sem informação.

Quanto à atividade religiosa familiar, mais da metade das famílias estudadas não frequentavam nenhuma atividade religiosa $(55,6 \%)$.

A configuração das famílias abarcadas neste estudo constitui-se de 46,8\% de famílias monoparentais e 53,2\% de biparentais, sendo que a maioria das crianças e adolescentes $(84,9 \%)$ estava sob a guarda dos genitores e $15,1 \%$ com outros guardiões legais.

Quanto à caracterização da violência doméstica, observou-se que os principais agressores eram a mãe $(42,9 \%)$ e os pais - mãe e pai $(33,3 \%)$, com menor frequência eram: apenas o pai $(13,9 \%)$, o padrasto/madrasta/ tio/tia (4,4\%), avô/avó (2,0\%) e na categoria outros, sendo guardiões/cafetina/outro adolescente/ vizinho (7,2\%); além disso, $7,1 \%$ das crianças e adolescentes sofreram diferentes tipos de violência praticada por mais de um autor, sendo a metade desses sujeitos vítimas de abuso sexual.

Verificou-se predominância de violência psicológica $(58,3 \%)$, que ocorreu associada a quase todos os tipos de violência e negligência $(56,7 \%)$, seguidas pela violência 
Hildebrand, N. A., Celeri, E. H. R. V., Morcillo, A. M. \& Zanolli, M. L. (2015). Violência Doméstica e Risco para Problemas de Saúde Mental em Crianças e Adolescentes.

física $(43,7 \%)$ e sexual (19\%). Além disso, $86,2 \%$ dos sujeitos eram vítimas de pelo menos dois ou mais tipos; e também, $10,7 \%$ das crianças e adolescentes estudados sofreram exploração do trabalho infantil.

É importante ressaltar que $\mathbf{9 6 , 8 \%}$ dos responsáveis pelas crianças e adolescentes estudadas foram vítimas de violência doméstica na infância e juventude, reproduzindo, assim, esse ciclo.

A possibilidade de prevalência de problemas de saúde mental foi de $65,5 \%$ (IC95\%, 59,3\% - 71,3), sendo que os sujeitos do gênero masculino apresentaram $66,4 \%$ (IC95\%, 57,2 - 74,8) e os do feminino, 64,7\% (IC95\%, 55,9 - 72,7; $p=0,774)$. Na comparação dos grupos formados, de acordo com a faixa etária, verificou-se maior prevalência de possibilidade de dificuldades na faixa etária de 8 a 11 anos (73,5\%, IC95\% 62,7 - 82,6), seguida pela dos adolescentes de 12 a 16 anos, com 65,4\% (IC95\%, 55,6 - 74,4). Destacase que $54,8 \%$ (IC95\%, 41,7 - 67,5) das crianças na faixa etária de 4 a 7 anos já apresentaram possibilidades de problemas de saúde mental. Não houve diferença estatística quanto à prevalência dos grupos etários $(p=0,065)$.

Quanto aos sintomas apresentados nas quatro escalas de problemas de saúde mental do SDQ, verificou-se que na sua maioria, as crianças e adolescentes apresentaram mais de um sintoma, sendo os mais frequentes os problemas de conduta $(66,3 \%)$, seguidos pelos problemas emocionais
(54,4\%) e de relacionamento (52\%) e, por último, atenção/ hiperatividade $(45,6 \%)$. Na subescala de comportamento pró-social, apenas $14,3 \%$ apresentaram dificuldades na superação dos problemas de ordem emocional.

Em relação aos possíveis fatores de risco para problemas de saúde mental verificou-se que $92,8 \%$ dos sujeitos desse estudo apresentaram exposição a pelo menos um deles, sendo que $53,2 \%$ estavam presentes dois ou mais fatores de risco associados. As frequências encontradas em cada um dos fatores estudados foram de: $42,9 \%$ de conflitos familiares entre os responsáveis $(p=0,541) ; 30,6 \%$ de problemas de saúde mental na família $(p=0,905) ; 26,2 \%$ de violência de gênero entre os pais $(p=0,323) ; 19,4 \%$ de uso preocupante de SPA $(p=0,978) ; 15,5 \%$ de envolvimento familiar com o tráfico de drogas/criminalidade $(p=0,574)$; $15,5 \%$ de uso preocupante de bebida alcóolica dos responsáveis $(p=0,018) ; 10,3 \%$ evasão escolar dos adolescentes ( $p=0,389) ; 9,9 \%$ de doença crônica ou questões de saúde na família $(p=0,870) ; 8,3 \%$ de ameaça de morte de alguém da família $(p=0,187) ; 6,7 \%$ de acolhimento institucional, quando a criança ou adolescente já foi abrigado em algum momento da vida $(p=0,323) ; 6,7 \%$ de gravidez na adolescência $(p=0,323) ; 4,4 \%$ de alguém na família estar foragido ou recluso $(p=0,752) ; 2,8 \%$ de situação de rua/ mendicância da família $(p=1,0) ; 2,4 \%$ de prostituição dos responsáveis $(p=0,185)$ e $0,4 \%$ de adolescentes em situação de medida socioeducativa $(p=1,0)$.

Tabela 1

Frequência na Amostra Estudada da Presença de Possiveis Fatores de Risco para Problemas de Saúde Mental em Crianças e Adolescentes Vítimas de Violência Doméstica

\begin{tabular}{lccc}
\hline Fatores de Risco & $f$ & $\%$ & $p^{*}$ \\
\hline Conflitos Familiares entre os responsáveis & 108 & 42,9 & 0,541 \\
Problemas de Saúde Mental na Família & 77 & 30,6 & 0,905 \\
Violência de Gênero entre os pais & 66 & 26,2 & 0,323 \\
Uso Preocupante de SPA** (alguém da família) & 49 & 19,4 & 0,978 \\
Envolvimento Familiar com Tráfico de Drogas/ Criminalidade & 39 & 15,5 & 0,574 \\
Uso Preocupante de Bebida Alcoólica dos Responsáveis & 39 & 15,5 & 0,018 \\
Evasão Escolar (adolescentes) & 26 & 10,3 & 0,389 \\
Doença Crônica ou Questões de Saúde na Família & 25 & 9,9 & 0,870 \\
Ameaça de Morte (alguém da família) & 21 & 8,3 & 0,187 \\
Acolhimento Institucional** & 17 & 6,7 & 0,323 \\
Gravidez na Adolescência & 17 & 6,7 & 0,323 \\
Foragido ou Recluso (alguém da família) & 11 & 4,4 & 0,752 \\
Situação de Rua/ Mendicância da Família & 07 & 2,8 & 1,0 \\
Prostituição dos Responsáveis & 06 & 2,4 & 0,185 \\
Medida Socioeducativa (adolescentes) & 01 & 0,4 & 1,0 \\
\hline
\end{tabular}

Notas. $p^{*}$ - Probabilidade, ${ }^{* *}$ SPA - Substâncias Psicoativas, ${ }^{* * *}$ Criança ou adolescente já foi abrigado em algum momento da vida.

Os fatores de risco o "Uso Preocupante de Bebida Alcoólica dos Responsáveis" $(p=0,018)$ e a "Inserção no Processo Produtivo" nas categorias "Não Trabalha/Desempregados e Aposentados/Recebem BPC" $(p=0,04)$ foram as- sociados à possibilidade de problemas de saúde mental nas crianças e adolescentes. Não foram encontradas associações entre as demais variáveis de fatores de risco e a possibilidade de problemas de saúde mental nos sujeitos estudados. 
Quando aos possíveis fatores de proteção, apesar de todos os sujeitos deste estudo estarem inseridos no atendimento especializado do CREAS, com tempo médio de acompanhamento de dois anos e meio (Desvio Padrão: 1,3) e o fato de que $\mathbf{9 6 , 4 \%}$ das famílias frequentassem e tinham como referência pelo menos um dos serviços da Rede de Proteção, verificou-se que frequentar tais serviços não foi fator de proteção para evitar a possibilidade de desenvolvimento de problemas de saúde mental nas crianças e adolescentes estudados. A frequência encontrada de sujeitos que contavam com os serviços da Rede de Proteção foi de $65,5 \%(p=0,788)$, para aqueles que tinham a educação como referência; $51,6 \%(p=0,275)$, a saúde; $43,3 \%(p=0,526)$, os SGD; 38,5\% $(p=0,494)$, os serviços de assistência social básica; $12,7 \%(p=0,526)$, os serviços de cultura, esporte, habitação, trabalho e renda (agrupados para análise).

Ainda em relação aos possíveis fatores de proteção, verificou-se que frequentar atividades socioculturais, esportivas ou comunitárias também não evitou o possível desenvolvimento de problemas de saúde mental nos sujeitos estudados, pois não foi encontrada associação entre participar dessas atividades e não apresentar possibilidades de psicopatologias, tanto no que diz respeito às crianças e adolescentes $(p=0,730)$, quanto às famílias $(p=0,775)$. Sobre a participação dos sujeitos nesses programas, apenas $38,5 \%$ das crianças/adolescentes e 35,3\% dos responsáveis o faziam. Quanto ao restante, que não participava desse tipo de atividade, $38,9 \%$ de crianças e adolescentes e 40,2\% das famílias apontaram a televisão como principal fonte de lazer. A falta de opção de programas culturais, sociais ou de diversão pode ser um risco potencial.

O fato de o responsável estar inserido em atividades produtivas nas categorias Trabalho Formal/Outros (Estagiários e Autônomos) e Trabalho Informal $(p=0,04)$ aparece associado à ausência de possíveis problemas de saúde mental nos sujeitos estudados. Não foi observada associação entre ausência de possíveis problemas de saúde mental e outros de fatores de proteção, como: frequentar atividades religiosas $(p=0,067)$, escolaridade dos responsáveis $(p=0,385)$ e morar com guardiões legais para proteção contra situações de violência $(p=0,486)$.

\section{Discussão}

Este estudo mostrou alta prevalência $(65,5 \%)$ de possíveis problemas de saúde mental em crianças e adolescentes vítimas de violência doméstica, atendidas em Serviços Especializados de Referência, de um município brasileiro, em relação à população geral (Ministério da Saúde, 2005; WHO, 2005). Esta prevalência é semelhante a dados encontrados em outros trabalhos da literatura com sujeitos vítimas de violência (Lorion \& Saltzman, 1993; Serafim et al., 2011). No caso da população estudada, a violência doméstica foi um risco potencial para o desenvolvimento de possíveis problemas psicoafetivos e psicopatológicos.
Ao analisar a prevalência de problemas de saúde mental em relação às faixas etárias, deve-se destacar o índice bastante elevado já nas crianças de 4-7 anos (54,8\%). Isso aponta para a necessidade de intervenções efetivas e específicas, com o objetivo de prevenir o desenvolvimento de transtornos mais graves. Observou-se que a faixa etária de 8-11 (73,5\%) apresentou aumento em relação à anterior, mas na faixa etária de 12-16 anos $(65,4 \%)$ houve diminuição. De acordo com as informações coletadas junto aos profissionais que acompanhavam as famílias, os adolescentes com maior risco para transtornos mentais apresentavam dificuldade em aderir aos atendimentos dos Serviços Especializados e também não estavam frequentando outros serviços de tratamento, proteção ou prevenção. Além disso, eles não compareceram às entrevistas para aplicação dos questionários, tornandose necessário substituí-los. Pressupõe-se, a partir desta análise, que, por estarem mais tempo expostos a situações adversas, os adolescentes seriam mais vulneráveis aos problemas de saúde mental (A. Goodman et al., 2007). E, com base nessa reflexão, seria importante que os serviços pudessem analisar a efetividade de sua atuação junto à população dessa faixa etária, visando a outras formas de abordagem, para garantir mais adesão pelos adolescentes a esse acompanhamento.

Outros dados que reafirmam essas suposições foram: com o aumento da idade dos sujeitos, obteve-se crescimento no número de crianças e adolescentes em atendimento nos Serviços Especializados e, consequentemente, no número de notificações em relação à violência doméstica. Pressupõe-se que, com seu amadurecimento, a criança passe a perceber e manifestar, nos diversos ambientes que frequenta, cada vez mais sinais e sintomas, como consequência da violência sofrida, ocasionando, assim, suspeitas/diagnósticos e notificações.

Os sintomas mais frequentes apresentados pelos sujeitos desse estudo foram problemas de conduta $(66,3 \%)$. Os emocionais $(54,4 \%)$, que se referem principalmente a sintomas de depressão e ansiedade e os de dificuldades nos relacionamento $(52 \%)$ foram semelhantes e, por último, os problemas de atenção, concentração e hiperatividade $(45,6 \%)$. Os transtornos psiquiátricos em crianças e adolescentes podem ocorrer por vários fatores. É fator a se considerar, que tem sido verificada associação entre problemas de comportamento ou conduta e variáveis do ambiente familiar, como a violência doméstica (Assis et al., 2009). Nesse sentido, os dados encontrados neste estudo corroboram os resultados da literatura.

Além disso, destaca-se que as crianças e adolescentes estudados apresentaram mais de um sintoma, indicando possibilidade de comorbidades em relação aos problemas de saúde mental, ocorrência comum em psiquiatria da infância e adolescência. Identificá-las ainda em etapas iniciais do desenvolvimento é fundamental, pois quando não tratadas podem se agravar, tornando-se mais complexas. Por outro lado, aumenta também a dificuldade de sensibilizar o sujeito, principalmente o jovem, sobre 
Hildebrand, N. A., Celeri, E. H. R. V., Morcillo, A. M. \& Zanolli, M. L. (2015). Violência Doméstica e Risco para Problemas de Saúde Mental em Crianças e Adolescentes.

a importância do tratamento, como pode ser confirmado pelos altos índices de absenteísmo entre os adolescentes (Assis et al., 2009).

Quanto à caracterização da população estudada em relação aos tipos de violência, verificou-se que a maioria das crianças e adolescentes sofreu pelo menos dois ou mais tipos diferentes concomitantemente, em mais de um episódio. Esses dados podem ser confirmados por pesquisa (Bazon, 2008) que mostra a ocorrência associada dos diferentes tipos de maus tratos. Como a violência psicológica mostrou-se presente em grande parte dos casos, pode ser apontada como um sinalizador para futuros problemas de saúde mental na vida adulta desses sujeitos (Abranches \& Assis, 2011).

Quase metade das famílias deste estudo eram monoparentais, compostas principalmente pela genitora como única responsável. Esse aspecto pode estar relacionado aos achados desta pesquisa, que indicaram a mãe como principal agressora, como também ao fato de a maioria das famílias estudadas apresentarem histórico de violência intrafamiliar transgeracional, ou seja, os responsáveis também serem vítimas de violência doméstica (Bérgamo \& Bazon, 2011; Walker, 1979). Pode-se pressupor que assumir responsabilidades familiares, socioeconômicas e educativas de um filho, sozinha, torna-se um fator de mais vulnerabilidade da mãe, uma vez que essa situação pode ser vivenciada por ela como ausência de apoio, principalmente quando associada ao histórico de violência doméstica dessas genitoras (American Psychological Association, 2003; Bérgamo \& Bazon, 2012).

Esses dados são condizentes com a literatura que aponta como possíveis fatores de risco para o desenvolvimento de problemas de saúde mental situações como: baixa renda familiar, baixa escolaridade dos responsáveis, violência comunitária, conflitos intrafamiliares, envolvimento dos responsáveis com a criminalidade, punição física severa, aspectos psicológicos dos cuidadores, o fato de ser órfão, vivenciar processo de institucionalização precoce e/ou prolongada e condições precárias de saúde na infância e adolescência. Todos esses fatores podem significar alto risco para o comprometimento do desenvolvimento psicoafetivo de crianças e adolescentes (Baptista, Soares, \& Henriques, 2013; Bérgamo \& Bazon, 2012; A. Goodman et al., 2007; Koller \& Lisboa, 2007; Poletto et al., 2009).

Ao analisar os possíveis fatores de risco, foram encontrados, como agravantes, o uso preocupante de bebida alcoólica dos responsáveis e sua situação trabalhista, nas categorias não trabalham por opção, desempregados, aposentados e beneficiários do BPC.

A população estudada, em sua totalidade apresenta alto risco social, pois quase todos os sujeitos deste estudo $(92,8 \%)$ foram expostos a, pelo menos, um fator de risco, e $53,2 \%$ a dois ou mais. Dessa forma, identificou-se que tanto aqueles do grupo de crianças/adolescentes que apresentaram problemas de saúde mental, quanto os que não apresentaram, foram expostos a inúmeras situações de vulnerabilidade. Os fatores de risco funcionam como agravantes para o desenvolvimento de problemas de saúde mental nos sujeitos estudado, uma vez que a violência doméstica, por si só, constitui-se como o principal risco.

A literatura aponta que os fatores de proteção têm as funções de: reduzir o impacto dos riscos, alterando, assim, a exposição da pessoa a situações adversas; minimizar as reações negativas, que podem surgir em cadeia, após a exposição do indivíduo a eventos estressores; estabelecer e manter a autoestima e autoeficácia, por meio de estabelecimento de relações vinculares afetivas e seguras, que facilitem o cumprimento de tarefas com sucesso; e criar oportunidades para reverter ou reparar os danos causados (Noronha, Cardoso, Moraes, \& Centa, 2009). Entretanto, neste estudo, os fatores de proteção analisados não se mostraram efetivos para evitar o desenvolvimento de problemas de saúde mental nos sujeitos estudados, com exceção da situação trabalhista dos responsáveis, quando esses estavam inseridos no processo produtivo.

A situação trabalhista dos responsáveis pelos sujeitos estudados pode ser analisada tanto como um indicador de risco em relação ao desemprego, quanto de proteção, quando esses responsáveis estavam inseridos no processo produtivo. Em estudo sobre condições de vida e estrutura ocupacional, indivíduos em situação de exclusão do mercado formal de trabalho apresentaram maior prevalência associada a transtornos mentais comuns (Ludermir \& Melo, 2002).

Nesse caso, pode-se pressupor que o trabalho proporciona algumas possibilidades potenciais ao sujeito, como melhores condições de renda, além de estabelecer e manter a autoestima e o sentimento de autoeficácia dos responsáveis. Depreende-se, neste estudo, que possa ter ocorrido diminuição em relação às situações de violência doméstica, devido às melhores condições relativas à qualidade de vida e à saúde mental, apesar desse dado não ter sido analisado estatisticamente.

Quando estudadas as outras variáveis (renda familiar e frequência em atividades sociais, comunitárias ou religiosas) como possíveis fatores de proteção, verificou-se um quadro preocupante, pois grande parte da população estudada não frequenta atividades socioculturais, nem religiosas, e a televisão foi apontada por grande parte dos sujeitos estudados como a principal fonte de lazer. A falta dessas atividades destaca-se como possíveis fatores de risco, em função da ausência de suportes externos para o desenvolvimento de potencialidades (Rutter, 1987; Werner, 1989).

Com relação à Rede de Proteção, verificou-se ausência na efetividade das políticas públicas de atendimento, pois não houve associação entre ausência de problemas de saúde mental e frequentar tais serviços. A maioria da população estudada encontra-se assistida pela saúde, educação, assistência social, cultura, esporte e o SGD. Pode-se inferir que, apesar de essas famílias estarem sendo acompanhadas por profissionais das diversas áreas, existe a necessidade de refletir acerca de estratégias metodológicas mais efetivas. 
Outro estudo (Rossetti-Ferreira et al., 2012) realizado com crianças vítimas de violência doméstica verificou resultados semelhantes, apontando para a necessidade de capacitação dos vários serviços e profissionais que atuam na Rede de Proteção infanto-juvenil. A efetividade de ações em rede possibilita o desenvolvimento integral de crianças e adolescentes em situações de abandono e violência, atendendo tanto aos seus direitos como aos de suas famílias.

As famílias deste estudo estão inseridas em Serviços Especializados, que têm como objetivo minimizar situações de VDCCA, por meio do acompanhamento psicossocial familiar. Entretanto, em relação aos possíveis problemas de saúde mental nas crianças e adolescentes acompanhados, por não ser foco deste estudo, não se pode afirmar que haveria, ou não, diminuição na prevalência dessas questões, tanto durante o processo de atendimento, ou mesmo após o desligamento desses sujeitos.

Em relação aos 35,5\% de sujeitos que não apresentaram risco para desenvolvimento psicoafetivo segundo o SDQ, pode-se pressupor que apresentam maior capacidade de resiliência do que àqueles que apresentaram possibilidades de transtornos mentais (Noronha et al., 2009; Rutter, 1985, 1987). O SDQ também apontou alta frequência de sujeitos com boa capacidade pró-social para o enfrentamento de eventos traumáticos $(85,7 \%)$. Tais situações, associadas à ausência de fatores de suportes externos, sinalizam para a necessidade de estudos mais aprofundados acerca de mecanismos protetivos e de promoção de resiliência, que poderiam contribuir para projetos preventivos e de intervenção mais efetivos e cientificamente balizados (Kotliarenco, Cárcares, \& Fontecilla, 1997). Quando o sujeito pode contar com uma rede de apoio efetiva, com reais possibilidades de mudanças, e adquire a percepção de que é capaz de superar as situações adversas vivenciadas, ampliam-se suas possibilidades de desenvolvimento emocional.

\section{Conclusão}

A violência doméstica vivenciada pelas crianças e adolescentes, deste estudo, é um fator de risco significativo para possibilidades de problemas de saúde mental na infância e juventude, agravado pelo uso preocupante de bebida alcoólica pelo responsável e/ou por este estar fora do processo produtivo.

A ausência de fatores de proteção efetivos, além da situação trabalhista do responsável, dificulta o desenvolvimento e a promoção da resiliência ou de capacidade do indivíduo desenvolver respostas saudáveis de enfrentamento à violência doméstica e às suas consequências.

Por serem crianças e adolescentes em seguimento em Centro de Referência, é indispensável que sejam avaliadas a efetividade do acompanhamento e a dificuldade de adesão ao tratamento, tanto delas, como de suas famílias. Para garantir a integralidade do cuidado e o avanço na concretização dos direitos desses sujeitos, também se faz necessário rever a efetividade das políticas públicas de enfrentamento à violência, de prevenção e de tratamento especializado em saúde mental para as crianças e adolescentes vítimas de violência doméstica. Nesse caso, é fundamental a articulação intrassetorial e intersetorial em rede, para integrar todas essas ações. Investimento em estudos sobre a resiliência e formas efetivas de proteção poderão contribuir para minimizar os efeitos emocionais decorrentes de situações de violência.

\section{Referências}

Abranches, C. D., \& Assis S. G. (2011). A (in) visibilidade da violência psicológica na infância e adolescência no contexto familiar. Cadernos de Saúde Pública, 27(5), 843-854. doi:10.1590/S0102-311X2011000500003

American Psychological Association. (2003). Advocate: The child, youth, and family services. Bulletin of the Division 37. Washington, DC: Author.

Assis, G. S., Avanci, J. Q., Pesce, R. P., \& Ximenes, L. F. (2009). Situação de crianças e adolescentes brasileiros em relação à saúde mental e à violência doméstica. Ciência e Saúde Coletiva, 14(2), 349-361. doi:10.1590/S1413-81232009000200002

Azevedo, M. A., \& Guerra V. N. A. (2006). Violência doméstica contra crianças e adolescentes: Um cenário em desconstrução. In Fundo das Nações Unidas para a Infância, Direitos negados: A violência contra a criança e o adolescente no Brasil. Brasília, DF: Fundo das Nações Unidas para a Infância. Recuperada em http://www.unicef.org/brazil/pt/Cap_01.pdf

Baptista, J., Soares, I., \& Henriques M. (2013). Recuperação desenvolvimental após a adoção: Características da criança e da família adotiva. Psicologia: Reflexão e Crítica, 26(2), 396-404. doi:10.1590/S0102-79722013000200020

Bazon, M. R. (2008). Violências contra crianças e adolescentes: Análise de quatro anos de notificações feitas ao Conselho Tutelar na cidade de Ribeirão Preto, São Paulo, Brasil. Cadernos de Saúde Pública, 24(2), 323-332. doi:10.1590/ S0102-311X2008000200011

Bérgamo, L. P. D., \& Bazon, M. R. (2011). Experiências infantis e risco de abuso físico: Mecanismos envolvidos na repetição da violência. Psicologia: Reflexão e Crítica, 24(4), 710-719. doi:10.1590/S0102-79722011000400011

Bérgamo, L. P. D., \& Bazon, M. R. (2012). Abuso físico infantil: Avaliando fatores de risco psicológicos em cuidadores notificados. Psicologia: Reflexão e Crítica, 25(2), 256-264. doi:10.1590/S0102-79722012000200007

Canino, G., Shrout, P. E., Rubio-Stipec, M., Bird, H. R., Bravo, M., Ramírez, R., ...Martinez-Taboas, A. (2004). The DSM-IV rates of child and adolescent disorders in Puerto Rico: Prevalence, correlates, service use, and the effects of impairment. Archives of General Psychiatry, 61(1), 85-93. doi:10.1001/ archpsyc.61.1.85

Cicchetti, D., \& Valentino, K. (2006). An ecological-transactional perspective on child maltreatment: Failure of the average expectable environment and its influence on child development. In Developmental Psychopathology: Vol. 3. Risk, disorder, and adaptation (pp. 129-201). New York: John Wiley \& Sons.

Day, V. P., Telles, L. E. B., Zoratto, P. H., Azambuja, M. R. F., Machado, D. A., Silveira, M. B., ...Blank, P. (2003). Violência doméstica e suas diferentes manifestações. Revista de Psiquiatria do Rio Grande do Sul, 25(1), 9-21. doi:10.1590/ S0101-81082003000400003 
Hildebrand, N. A., Celeri, E. H. R. V., Morcillo, A. M. \& Zanolli, M. L. (2015). Violência Doméstica e Risco para Problemas de Saúde Mental em Crianças e Adolescentes.

Duarte, C. S., \& Bordin, I. A. S. (2000). Instrumentos de avaliação. Revista Brasileira de Psiquiatria, 22(2), 55-58. doi:10.1590/S1516-44462000000600015

Fleitlich-Bilyk, B., Cortazar, P. G., \& Goodman, R. (2000). Questionário de Capacidades e Dificuldades (SDQ). Infanto, Revista de Neuropsiquiatria da Infância e Adolescência, 8 , 44-50.

Fleitlich-Bilyk, B., \& Goodman, R. (2001). Social factors associated with child mental health problems in Brazil: Cross sectional survey. Britsh Medical Journal, 323, 599-600. doi:10.1136/bmj.323.7313.599

Fleitlich-Bilyk, B., \& Goodman, R. (2004). Prevalence of child and adolescent psychiatric disorders in southeast Brazil. Journal of the American Academy of Child Adolescent Psychiatry, 43, 727-734. doi:10.1097/01.chi.0000120021.14101.ca

Gallo, E. A. G., Anselmi, L., Dumith, S. C., Scazufca, M., Menezes, A. M. B., Hallal, P. C., \& Matijasevich, A. (2011). Tamanho ao nascer e problemas de saúde mental aos 11 anos em uma coorte brasileira de nascimentos. Cadernos de Saúde Pública, 27 (8), 1622-1632. doi:10.1590/S0102$-311 \mathrm{X} 2011000800017$

Goodman, A., Fleitlich-Bilyk, B., Patel, V., \& Goodman, R. (2007). Child, family, school and community risk factors for poor mental health in Brazilian Schoolchildren. Journal of the American Academy of Child and Adolescent Psychiatry, 46(4), 448-456. doi:10.1097/chi.0b013e31803065b5

Goodman, R. (1997). The Strengths and Difficulties Questionnaire: A research note. Journal of Child Psychology and Psychiatry, 38, 581-586. doi:10.1111/j.1469-7610.1997. tb01545.x

Goodman, R. (2001). Psychometric properties of the Strengths and Difficulties Questionnaire. Journal of the American Academy of Child and Adolescent Psychiatry, 40, 1337-1345. doi:10.1097/00004583-200111000-00015

Koller, S. H., \& Lisboa, C. (2007). Brazilian approaches to understanding and building resilience in at-risk populations Child and Adolescent Psychiatric Clinics of North America, 6(2): 341-356. doi:10.1016/j.chc.2006.12.002

Kotliarenco, M. A., Cáceres, I., \& Fontecilla, M. (1997). Estado de arte en resiliencia. Washington, DC: Organización Panamericana de la Salud. Recuperado em http://www.ugr. es/ javera/pdf/2-3-resiliencia\%20libro.pdf

Lorion, R. P., \& Saltzman, W. (1993). Children's exposure to community violence: Following a path from concern to research to action. Psychiatry, 56(I), 55-65.

Ludermir, A. B., \& Melo, D. A., Filho. (2002). Condições de vida e estrutura ocupacional associadas a transtornos mentais comuns. Revista de Saúde Pública, 36(2), 213-221. doi:10.1590/ S0034-89102002000200014

Ministério do Desenvolvimento Social e Combate à Fome. (2005). Sistema Único de Assistência Social (SUAS, Norma Operacional Básica - NOB/SUAS) - Construindo as bases para a implantação do Sistema Único de Assistência Social. Brasília, DF: Editora do Ministério da Saúde. Recuperado em http://www.mds.gov.br/assistenciasocial/arquivo/normaoperacional-basica-do-suas.pdf/view

Ministério do Desenvolvimento Social e Combate à Fome. (2010). Sistema Único de Assistência Social (SUAS): Manual informativo para jornalistas, gestores e técnicos. Brasília, DF: Editora do Ministério da Saúde. Recuperado em http:// www.mds.gov.br/assistenciasocial/secretaria-nacional-deassistencia-social-snas/guias/sistema-unico-de-assistenciasocial-suas-manual-informativo-para-jornalistas-gestores-e- tecnicos/sistema-unico-de-assistencia-social-suas-manualinformativo-para-jornalistas-gestores-e-tecnicos

Ministério da Saúde. (2005). Caminhos para uma politica de saúde mental infanto-juvenil. Brasília, DF: Autor. Recuperado em http://bvsms.saude.gov.br/bvs/publicacoes/05_0887_M.pdf

Ministério da Saúde. (2008). Impacto da violência na saúde das crianças e adolescentes: Prevenção de violências e promoção da cultura de paz. Brasília, DF: Autor. Recuperado em http:// www.feim.org.ar/pdf/blog_violencia/afc_cartilha_a5b.pdf

Noronha, M. G., Cardoso, P. S., Moraes, T. N. P., \& Centa, M. L (2009). Resiliência: Nova perspectiva na promoção da saúde da família? Ciência e Saúde Coletiva, 14(2), 497-506. doi:10.1590/S1413-81232009000200018

Paula, C. S., Duarte, C. S., \& Bordin, I. A. S. (2007). Prevalence of mental health problems in children and adolescents from the outskirts of Sao Paulo City: Treatment needs and service capacity evaluation. Revista Brasileira de Psiquiatria, 29(1), 11-17. doi:10.1590/S1516-44462006005000012

Paula, C. S., Vedovato, M. S., Bordin, I. A., Barros, M. G. S. M., D’Antino, M. E. F., \& Mercadante, M. T. (2008). Saúde mental e violência entre estudantes da sexta série de um município paulista. Revista de Saúde Pública, 42(3), 524-528. doi:10.1590/S0034-89102008005000027

Polanczyk, G. V., Zavaschi, M. L., Benetti, S., Zenker, R., \& Gammerman, P. W. (2003). Violência sexual e sua prevalência em adolescentes de Porto Alegre, Brasil. Revista de Saúde Pública, 37(1), 8-14. doi:10.1590/S0034-89102003000100004

Poletto, M., Koller, S. H., \& Dell'Aglio, D. D. (2009). Eventos estressores em crianças e adolescentes em situação de vulnerabilidade social de Porto Alegre. Ciência e Saúde Coletiva, 14(2), 455-466. doi:10.1590/S1413-81232009000200014

Rossetti-Ferreira, M. C., Almeida, I. G., Costa, N. R. A., Guimarães, L. A., Mariano, F. N., Teixeira, S. C. P., \& Serrano, S. A. (2012). Acolhimento de crianças e adolescentes em situações de abandono, violência e rupturas. Psicologia: Reflexão e Crítica, 25(2), 390-399. doi:10.1590/S010279722012000200021

Rutter, M. (1985). Resilience in the face of adversity: Protective factors and resistance to psychiatric disorder. British Journal of Psychiatric, 147(6), 598-611. doi:10.1192/bjp.147.6.598

Rutter, M. (1987). Psychosocial resilience and protective mechanisms. American Journal of Orthopsychiatry, 57(3), 316-331. doi:10.1111/j.1939-0025.1987.tb03541.x

Serafim, A. P., Saffi, F., Achá, M. F. F., \& Barros, E. M. (2011). Dados demográficos, psicológicos e comportamentais de crianças e adolescentes vítimas de abuso sexual. Revista de Psiquiatria Clínica, 38(4), 143-147. doi:10.1590/S010160832011000400006

Walker, L. (1979). The battered woman. New York: Harper and Row.

Werner, E. E. (1989). High-risk children in young adulthood: A longitudinal study from birth to 32 years. American Journal Orthopsychiatric, 59(1), 72-81. doi:10.1111/j.1939-0025.1989. tb01636.x

Word Health Organization. (1999). Report of the consultation on child abuse prevention. Geneva, Switzerland: Author.

Word Health Organization. (2005). Child and adolescent Atlas. Resources for child and adolescent mental health. Geneva, Switzerland: Author. 